\title{
Aprendizaje Colaborativo en un módulo de formación docente basado en Blended Learning
}

\author{
Ilich Silva-Peña*, Isabel Salgado-Labra, Cristián Verdugo, \\ Ana Chehuaicura
}

\begin{abstract}
Resumen
El presente artículo nos entrega los resultados de la incorporación de herramientas para el aprendizaje colaborativo en un módulo de Formación Inicial Docente a través del sistema Blended Learning. Para el desarrollo de la experiencia se utilizó software con características Open source. En cuanto a la recogida de información, esta se realizó a través de la aplicación de cuestionarios con respuestas abiertas que fueron contestados por 12 estudiantes del curso. El análisis se realizó a través de un proceso de codificación que implicó utilizar el software Atlas Ti. Dentro de los principales hallazgos podemos señalar que la generación de un aprendizaje colaborativo en un ambiente Blended Learning está asociado al uso de la plataforma, las relaciones entre pares, las relaciones con la docente y el clima que se genera en la clase.
\end{abstract}

Palabras clave: Aprendizaje cooperativo, Blended Learning, Tecnología Educativa, Instrucción basada en Web.

\section{Collaborative learning in a teachers training module based on blended learning}

\begin{abstract}
This article presents the results of the incorporation of collaborative learning tools in a initial teachers training module based on the blended learning system, using an Open source software. Data collection was made through the application of open-ended questionnaires answered by $\mathrm{I} 2$ students. The analysis was made through a codification process using the Atlas Ti software. One of the main findings is the link between the development of a collaborative blended learning environment and the use of platforms, pair relationships, teacher-student relationship and classroom environment.
\end{abstract}

Key word: Collaborative Learning; Blended Learning; Education Technology; Web-based Teaching.

* Instituto de Investigaciones Pedagógicas de la Universidad Arturo Pratt.

E-mail: ilichsp@gmail.com 


\section{Introducción}

Una de las críticas que se ha formulado a las políticas educativas aplicadas en Chile, ha sido la existencia de debilidades en la formación docente, tanto inicial, como continua (OCDE, 2004). Dentro de este contexto se plantea la indagación sobre modelos de formación que incorporen las nuevas tecnologías bajo nuevas miradas del aprendizaje. El caso que se presenta se realizó en la Formación Inicial Docente, pero es posible realizar algunas transferencias a la Formación Continua.

Se plantea avanzar en la realización de mejoras en el apoyo pedagógico que reciben los estudiantes a través de esta modalidad de educación, para esto se considera la elaboración de modelos, sistemas y estrategias pedagógicas para la formación docente con características distintas a las tradicionales y que cumpla con los estándares de formación inicial.

Específicamente la investigación se planteó la construcción de un modelo de aprendizaje colaborativo dentro del proceso de formación inicial docente realizado en la Universidad Arturo Prat (Sede Victoria), bajo el concepto de Blended Learning o Blended Education que podemos definir a grandes rasgos como una educación que mezcla la educación presencial con aquella recibida a través de E-Learning (Motteram, 2006).

Para implementar dichas mejoras, se ha realizado una evaluación de distintas posibilidades de utilización de software, determinando utilizar Moodle por las características abiertas del programa, su constante renovación y la amplia información disponible en la red que apoya dicha plataforma.

\subsection{Aprendizaje colaborativo}

Podemos entender el aprendizaje colaborativo como "el uso instruccional de pequeños grupos de tal forma que los estudiantes trabajen juntos para maximizar su propio aprendizaje y el de los demás" (Johnson \& Johnson, citado en Collazos, Guerrero, \& Vergara, 200I), en este contexto, los docentes no dictan instrucciones específicas sino que permiten a los estudiantes elegir y variar sobre 
lo esencial de la clase y las metas a lograr, de este modo facilitan la participación de los estudiantes en su propio proceso de aprendizaje (Del Valle \& López, 2005).

Uno de los primeros estudios que señalan los beneficios del aprendizaje colaborativo es el de Amigues (1987), pero son Walberg y Paik (2000) quienes indican la existencia de más de 50 estudios que avalan que el aprendizaje resulta más eficaz cuando se utiliza este tipo de estrategias. Uno de los argumentos es que "cuando los alumnos trabajan en grupos de dos a cuatro componentes, cada miembro puede participar más y es más probable que los problemas individuales se puedan aclarar y resolver" (p. 16).

Otro elemento que se plantea en la construcción de ambientes de aprendizaje colaborativo es un cambio de rol en el/la docente, donde éste/a adquiere un carácter más propositivo que impositivo. En este aspecto, tal como señalan Collazos et al. (200I), los docentes invitan a sus estudiantes a definir los objetivos específicos dentro de la temática que se está enseñando, se entregan opciones y se les anima a que utilicen su propio conocimiento, asegurando de esta manera que lo compartan, como también que compartan sus estrategias de aprendizaje.

Según Collazos et al. (200I), no existen estudios que señalen en qué contextos específicos de aprendizaje colaborativo se producen los beneficios aludidos, por tanto se hace necesario avanzar en la comprensión de esos contextos, situación que se aborda este proyecto al tomar en cuenta un contexto mixto de enseñanza-aprendizaje en la formación inicial docente.

\subsection{Uso de las nuevas tecnologías en la formación inicial docente}

Casi no hay discusión acerca de que la incorporación de tecnologías de comunicación e información en la Formación Inicial Docente es de vital relevancia. Como ejemplo, en Chile se han realizado millonarias inversiones en el ámbito de la incorporación de tecnología en el aula (ver www.enlaces.cl), sin embargo la percepción de estudiantes sigue siendo que hay poca preparación por parte de los/as docentes (SilvaPeña, Borrero, Marchant, González y Novoa, 2006). 
En el último tiempo se han realizado diversas investigaciones que dan cuenta de experiencias de Blendend Learning, entendida como una mezcla entre el aprendizaje virtual y el presencial. Generalmente estas Investigaciones + Desarrollo + Innovación han estado en función de un módulo dentro de un programa, así podemos citar ejemplos realizados en Educación Secundaria (Yeh, Lee \& Sun, 2005), en pregrado (Bellefeuille, 2006) o clases de postgrado (Motteram, 2006).

Además de generar una importante información a la propia institución en cuanto al diseño del proceso mismo (Mutula, Kalusopa, Moahi, \& Wamukoya, 2006). En artículos publicados en el último tiempo se han dado a conocer otros resultados de dichas experiencias, las cuales apoyan la generación de comunidades naturales que además aportan en la transformación de los estudiantes en cuanto a la reflexión de su práctica día a día (Motteram, 2006). En el caso de experiencias de postítulo en profesores, se habla de un apoyo a la identidad profesional a partir de la constitución de comunidades de práctica (Guldberg \& Pilkington, 2006). En este sentido cobra relevancia el estudio de los resultados positivos que se producen en estudiantes y/o docentes, así como también en la constitución de la cultura que está involucrada en aquello.

\subsection{Contexto de la Experiencia}

Esta investigación se desarrolló en el contexto de una experiencia que incorpora una plataforma como apoyo al proceso de Formación Inicial Docente. Dentro de los módulos o cursos considerados en el sistema de formación docente existen algunos que se desarrollan con sistemas de tutorías, esto quiere decir que además de las clases presenciales se deben planificar sesiones "no presenciales". Las tutorías consisten en un trabajo organizado para el autoaprendizaje de los estudiantes guiados por el/la docente a cargo del curso. Generalmente estas tutorías corresponden a trabajos de profundización de las clases presenciales.

El propósito principal de esta experiencia era incorporar algunas prácticas de aprendizaje colaborativo utilizando una plataforma 


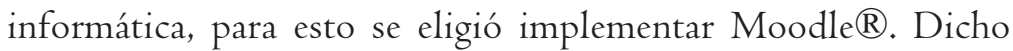
software posee las características de ser open source y de construcción colectiva, pero especialmente se optó por la flexibilidad del sistema y la mirada constructivista que lo sustenta. Esto nos animó a instalar la plataforma y utilizarla como prueba en diversos contextos y posteriormente usarla en un módulo de Formación Inicial Docente sobre Expresión Oral y Escrita destinado a estudiantes de segundo semestre de Pedagogía en Educación Básica de la Universidad Arturo Prat en la ciudad de Victoria (Chile).

Para la realización de la experiencia se organizó el trabajo con la docente, quien a su vez explicó a los/as estudiantes el trabajo de investigación que se iba a realizar, en este sentido, podemos decir que la disposición de la docente como de los/as estudiantes permitió avanzar en la experiencia de manera exitosa.

\section{Método}

La propuesta metodológica del presente estudio se enmarca dentro de los estudios cualitativos. La metodología cualitativa busca la descripción, la comprensión de las cualidades de un problema o pregunta, en este caso a través de las percepciones que tienen los/ as profesionales de la educación en formación. La idea principal es avanzar en estas opiniones más allá de la cuantificación del uso de la tecnología en Chile y revisar cuáles son las posibilidades de la implementación de este tipo de tecnologías en la Formación Inicial Docente en contextos específicos.

\subsection{Participantes}

Los participantes de la investigación fueron I2 estudiantes que participaron del curso de Expresión Oral y Escrita. El curso fue planteado como un curso con actividades de taller, las cuales se realizaban de modo no presencial; estas actividades eran de carácter paralelo a las actividades presenciales. Cabe señalar que los/as estudiantes eran de primer año de Pedagogía en Educación Básica, en el contexto de una modalidad vespertina, muchos/as de ellos/ as tenían otras actividades durante el día, como trabajo remunerado 
o labores domésticas. Provenientes de diferentes comunas de la provincia de Malleco, especialmente de la comuna de Victoria. No existía un acercamiento previo al uso de internet en algunos casos, razón por la que se comenzaron algunas sesiones con la creación de correo electrónico y uso de browser. En la plataforma se utilizaron principalmente Foros y Wikis durante el transcurso de la experiencia.

\subsection{Materiales}

Se utilizó una pauta para recoger información en los foros en los cuales participaron los estudiantes. La pauta permitió recoger los principales dichos referentes al aprendizaje colaborativo.

Además se aplicó un cuestionario de 10 preguntas de carácter abierto destinadas a recabar información para dar cuenta de los objetivos propuestos para la investigación. Se utilizaron los foros como una manera de recoger información acerca de las apreciaciones del curso y de la plataforma misma.

Se analizaron 5 foros y 12 cuestionarios, utilizando para ello el software Atlas Ti $\AA$, con el cual se construyeron redes que permitiesen establecer la relación entre los diferentes códigos extraídos a partir del primer análisis.

\subsection{Procedimientos}

El cuestionario fue aplicado de manera presencial durante una sesión especial dedicada a este objetivo. El análisis tanto de los cuestionarios como de los foros fueron realizados una vez terminado el curso, de este modo se pretendió evitar la influencia de la calificación como modo de presión para contestar el cuestionario.

Para el análisis se han utilizado nombres ficticios con el fin de salvaguardar el anonimato en la entrega de la información, pero manteniendo el relato coherente y que permita ayudar en la lectura del texto. 


\section{Resultados}

En esta sección se describirán los resultados obtenidos a partir del análisis realizado a los cuestionarios y foros. Se procede a reconstruir los distintos aspectos que forman parte del aprendizaje colaborativo en la experiencia señalada.

\subsection{Apoyo Docente}

En esta experiencia podemos observar que el apoyo docente se menciona más bien en relación a los aspectos técnicos más que a los contenidos.

El apoyo más importante fue que al formular una pregunta, ella nos contestaba de inmediato (Estudiante, Cuestionario 8, 6:6).

Que cuando uno le enviaba una consulta, la contestaba al tiro (Estudiante, Cuestionario II, II:5).

El apoyo del que dan cuenta los/as estudiantes está mediado por la plataforma, principalmente en la idea de utilizarla como un espacio para la comunicación y conexión entre los/as estudiantes. Cuando se refiere a "de inmediato" o "al tiro" nos da cuenta de una preocupación, una entrega de tiempo para la explicación otorgada por la docente, esta explicación si bien es cierto estaba focalizada en los aspectos técnicos, era una antesala para el trabajo que debían realizar.

\subsection{Interacción Docente-estudiante}

Hablamos de la interacción docente-estudiante como la relación que se establece a través de la plataforma durante el proceso enseñanzaaprendizaje, "no sólo se ponía delante de uno a enseñar sino que además esa enseñanza la llevábamos a la práctica en el aprendizaje” (Estudiante, Cuestionario 2, 4:4).

Se expresa la diferencia entre la enseñanza tradicional del profesor, se hace referencia a la teoría y ésta aplicada en la práctica. Esta visión

I Al tiro es una expresión particular utilizada en Chile, es sinónimo de inmediato. 
del proceso educativo parece ser más significativa o valorada por el estudiante.

Creo que tiene razón en cuanto al trato que existe entre docente y alumno, creo que debiera haber una relación más amena, como entregando una cierta confianza al alumno para que él se sienta en confianza con el docente y también encuentro muy buena la plataforma ya que, como lo dijo María, los mantiene a todos informados (Estudiante, Foro 3 4I:4I).

Se expone la situación de comunicación entre el alumno y el profesor, se analiza el aspecto de la confianza entregada, quizá se refiere a una relación de amistad. No se especifica a qué tipo de confianza se refiere. Sin embargo, es importante destacar que es un tema que se origina por la utilización de esta plataforma, es decir, se pueden expresar necesidades de comunicación que de otro modo no se darían.

Profesora, sin duda opino lo mismo en cuanto a la plataforma, me parece una excelente forma de mantener al curso comunicado, en relación a nosotros como curso y a usted como profesora (Estudiante, Foro 3 50:50).

Es notable la valoración que se le entrega a la plataforma como un apoyo para esta interacción pedagógica que se produce entre docente y estudiantes. La plataforma es vista como una forma de comunicación y conexión inter curso y entre el curso y la docente.

\subsection{Retroalimentación docente. Relación Docente - Alumno}

Como parte de la construcción de este aprendizaje colaborativo se encuentra la retroalimentación entre la docente y los/as estudiantes. Nos referimos a la retroalimentación como esta respuesta que realiza la docente a las interrogantes y cuestionamientos por parte de los/as estudiantes. En este sentido, podemos ver que existe una permanente preocupación por parte de la docente para reencuadrar la actividad en torno a los objetivos transmitidos en un comienzo: "el tema de este foro son tus habilidades y conocimientos en computación... creo que te confundiste" (Docente, Foro I 40:40). 
A veces, en un lenguaje bastante directo, la docente expone a sus alumnos y alumnas la necesidad de realizar un mejoramiento en sus trabajos: "Estimadas, creo que no se esforzaron mucho que digamos... ¿podrían ser más específicas respecto a lo que saben o no de este tema?” (Docente, Foro I 300:300).

La docente a cargo del curso también apoyaba anímicamente con distintas palabras que podrían ayudar al trabajo de las/os estudiantes: "gracias por tu sinceridad, ahora tenemos un buen desafío..." (Docente, Foro I 39I:39I).

En general en la relación docente-estudiante existió un lenguaje directo, con una preocupación por hacer más profundo el aprendizaje. La docente está preocupada por hacer avanzar a quienes dan muestra de una mayor actividad en el curso.

\subsection{Utilidad de la Plataforma}

Un elemento importante señalado por los/as estudiantes, es la utilidad que entrega la plataforma tecnológica. Los dichos de los participantes dan cuenta de las posibilidades para su vinculación en torno al curso mismo, pero también en torno a la conformación como curso, construcción de lazos como compañeros de carrera y de curso.

Porque mantiene a todo el curso unido relacionando y comparando temas de trabajo (Estudiante, Cuestionario 6, 8:8).

Profesora, sin duda opino lo mismo en cuanto a la plataforma, me parece una excelente forma de mantener al curso comunicado, en relación a nosotros como curso y a usted como profesora (Foro 3, Estudiante, 50:50).

La posibilidad de colaboración con otros es atribuida en parte a la plataforma, a la forma de vincularse a través de ella y las posibilidades que entrega de retroalimentación entre ellas. Esto de alguna manera corresponde a un trabajo en conjunto, ven como una posibilidad la retroalimentación de los escritos y de esta forma la construcción de aprendizajes de manera colectiva: "las ventajas según yo serían que si se tenía alguna duda no se interrumpía la clase de alguna manera y 
se podía hacer la pregunta a la profesora" (Estudiante, cuestionario $7,2: 2)$.

La plataforma permitía la posibilidad de ir más allá de la clase misma, de avanzar en los términos efectivamente planteados que era de la realización de aquellas actividades no presenciales, esto significaba que la clase se extendía "realmente" más allá de lo presencial. En este sentido la plataforma efectivamente cumplía con su rol de ser soporte del trabajo no presencial, un trabajo planificado, pero a la vez con espacios para lo no determinado que siempre surge en los procesos de aprendizaje-enseñanza:

En la plataforma se publicaba algún trabajo, que había que enviarlo dentro de un plazo: a la vez todos los participantes podían opinar o retroalimentar el escrito, así se producían aprendizajes entre todos. Porque mantiene a todo el curso unido relacionando y comparando temas de trabajo.

(Estudiante, Cuestionario 9, 4:4)

A través de ella pudimos entregar nuestros trabajos, redactar textos, escribir, comprensión lectora. Trabajamos con el sistema wiki, aprendimos a hacer resúmenes, integrar nuestras ideas al mismo tiempo que nuestros compañeros.

(Estudiante, Cuestionario I2, 5:6)

A continuación daremos cuenta de los elementos que son parte de esta apreciación positiva que se le otorga a la plataforma tecnológica utilizada. Cabe recordar que la plataforma (Moodle) no fue utilizada con todas sus herramientas, sino que básicamente se usaron los Foros, la entrega de tareas y los wikis.

\subsection{Uso de Foros}

Los Foros son considerados como una vía para la interacción entre los compañeros, como una manera de enviar comentarios a los trabajos de otros/as. La posibilidad de tener un espacio para compartir opiniones y comentarios sobre los temas del propio curso son elementos valorados por los/as estudiantes. Entre los elementos que son comunicados no sólo se incorporan aquellos solicitados por 
la docente, sino que también los que son parte de la constitución de grupo-curso, la constitución de comunidad de aprendizaje:

foros que sirvieron de medios para interactuar con los demás compañeros.

(Estudiante, Cuestionario 5, 7:7)

El foro, ya que todos tenían acceso a ellos y podían enviar su propio comentario.

(Estudiante, Cuestionario 7, 5:5)

Me parece bastante interesante la puesta en marcha de la plataforma. Sin duda un medio electrónico para manifestar no solamente nuestras inquietudes sino también para lo que nos sucede a veces como estudiantes.

(Estudiante, Foro 2, 253:253)

Aquí se establece una diferencia entre lo personal y lo académico, estimando que la plataforma es capaz de incorporar ambas dimensiones en un solo lugar. En este sentido la tecnología va en apoyo de la constitución de esta comunidad no solamente en torno a los contenidos y herramientas de aprendizaje sino que también en aquellos aspectos que hablan del clima interno, de las emociones transmitidas.

\subsection{Participación}

Tal como es señalado acerca de los foros en torno a posibilitar la comunicación, se aprecian las instancias de participación, el ser parte de un sistema, de una organización. Podemos decir entonces que también la plataforma es vista como un medio para afiatar los lazos de identidad y pertenencia a un determinado grupo.

Sí, y de mucho, ya que a ella todos los estudiantes que nos incorporamos al curso podíamos acceder, dando opiniones, comentarios, subiendo los trabajos, etc.

(Estudiante, Cuestionario 2, 9:9)

Poder ser parte de la página Instituto Pedagógico y poder realizar actividades en el laboratorio de computación.

(Estudiante, Cuestionario, 2:2) 


\subsection{Sitio Informal}

Se habilitó dentro de los foros, uno destinado a aspectos informales, como una especie de Off Topic del curso. La posibilidad de tener un espacio que no se limita solamente a los aspectos de contenido del curso es valorado por los estudiantes, aunque de todos modos permanece el recelo a la utilización de un lenguaje "demasiado informal”. Un ejemplo de lo anterior es cuando los estudiantes señalan que se pueden "escapar palabras indebidas". Aun considerando que el espacio "informal" es parte del curso, permite un cierto rango de libertad en la comunicación y la interacción al interior del grupo.

Este foro es para ingresar datos, novedades, comentarios, curiosidades, etc. La idea es que sea un espacio más informal de anuncios del curso.

(Docente, Foro 3, 12:12)

Enseñarnos a utilizar la plataforma y otros sitios para poder estar más en contacto con nosotros mismos y con ella por supuesto, aparte que teníamos un sitio que era informal y era bueno.

(Estudiante, Cuestionario 4, 6:6)

Creo que aunque sea informal es como raro porque a pesar de que sea así el hecho de que usted lo lea da cosa porque se pueden escapar palabras indebidas, uuuuuuuuuy pero en fin, es bueno.

(Foro 3, Estudiante, 29:29)

\subsection{Wiki}

El Wiki es un medio que permite un proceso de aprendizaje que incluye trabajo en equipo, enlace a través de una plataforma, lo que lo hace distinto, "la Wiki ya que nos permitió trabajar en equipo de una manera distinta, conectados a través de enlaces" (Estudiante, Cuestionario 4, 5:5).

El Wiki es una herramienta que podría permitir un mejor aprovechamiento, pero quizás no hay todavía suficiente apropiación de la tecnología. Es importante señalar que los participantes de la experiencia no tenían conocimiento previo en la realización de wikis, 
por tanto, el trabajo implementado en esta ocasión les permitió acercarse a esta nueva tecnología y utilizarla en su favor como un modo de realizar un trabajo colaborativo.

\subsection{Retroalimentación entre pares}

De alguna manera se genera una posición de apoyo desde otros estudiantes, es una forma a través de la cual se establece un proceso que hemos denominado como "retroalimentación entre pares", proceso en el que se crean lazos en función del proceso de aprendizaje, especialmente cuando hablamos de un proceso de aprendizaje de carácter colaborativo.

"Me parece súper interesante tu tema y qué bueno que lo hayas tomado" (Foro I, Estudiante, 26:26).

"Me parece interesante tu comentario y muy válida la preocupación que planteas...” (Foro I, Estudiante, 55:55).

"Como grupo lo hicieron muy bien!!!! Dejaron muy bien explicado los pasos a seguir para hacer una buena presentación.... y eso se agradece... xaito.” (Foro I, Estudiante, 30:30).

Los comentarios escritos promovían la creatividad y el mejoramiento de los niveles de desempeño del curso. La generación de un ambiente de apoyo en torno a las actividades propuestas por la docente y que eran parte del curso.

"En cuanto al comentario de los trabajos e investigaciones realizadas, nuestros compañeros criticaban de forma objetiva lo realizado para luego presentarlo de una mejor manera" (Estudiante, cuestionario 2, 10:10).

Aquí podemos observar que fueron considerados los comentarios realizados a través de la plataforma y que permitían este aprendizaje entre pares que es propio del aprendizaje colaborativo. Los comentarios por tanto, no solo tendrían como función entregar más seguridad en torno al trabajo que se estaba realizando, sino que también se entregaba un apoyo a la construcción de dicho trabajo. 
"Me parece bastante interesante la postura y el desarrollo de la mesa redonda. En realidad tenía otra sensación de cómo se estructuraba. Los tiempos de exposición y los espacios para conformar las preguntas son interesantes. Felicitaciones al grupo" (Foro I, Estudiante, 42:42).

"A ustedes les salió bien su mesa redonda, sobre todo a las compañeras, me dejaron claro de qué se trata una mesa redonda, el mediador también actúa bien. xao." (Foro I, Estudiante, 46:46).

"Tu texto es bastante pobre, no sé, en las ideas fundamentales del texto trabajado" (Foro 3, Estudiante, I53:153).

"Chicos, encuentro que su presentación estuvo buena, en un comienzo como que se confundió con el debate, pero luego quedó claramente entendido.... felicidades!!!!!" (Foro 5, Estudiante, 66:66)

"A mí igual me gustó el debate que presentaron, esta vez le salio súper bien sobre todo a la señora Mirta, a el Julio le faltó un poco más de discusión, pero se notó que era un debate muy interesante" (Foro 5, Estudiante, 86:86).

En algunas oportunidades la crítica va más allá de los aspectos formales de la exposición, la oratoria de la actividad, el desempeño de los participantes, y se hace un poco más profunda al realizar una evaluación cualitativa del desempeño. Se realiza la crítica especificando lo que no quedó claro de la exposición, aportando lo que se debe mejorar como una contribución al aprendizaje. Esto parece ser constructivo en el desarrollo personal y profesional de los estudiantes, constituyéndose una retroalimentación efectiva.

"Si esa es la idea, ayudarnos entre todos, pero por sobre todas las cosas, respetándonos con retroalimentación como tú dices; lo justo sería que como curso ayudáramos no sólo al Roberto sino a todo el que tenga alguna dificultad, cierto? (Foro I, Estudiante, 13:15).

"Súper de acuerdo, de hecho he formado grupo con él" (Foro I, Estudiante, 96:96). 


\subsection{Calificación}

La calificación ocupa un lugar importante a la hora de establecer las relaciones en el curso. Es un aspecto importante para la participación, así como para lo que dice relación con la realización de trabajos solicitados. Se establece una necesidad de integrarse e interrelacionarse en torno a la calificación, lo que es una motivación legítima e importante en un contexto en donde la evaluación significa no solo status sino que también beneficios económicos presentes y futuros. En ese sentido cobra relevancia el hecho de que existiera una tendencia a conseguir una buena calificación en el curso más allá del aprendizaje logrado.

"todos en una parte queríamos que nos fuera bien en nuestros ramos así que decidíamos conformar grupos con gente que creyéramos que serían potencia para cada calificación" (Foro I, Estudiante, I00:I0I).

\section{Conclusiones}

En primer lugar, podemos dar cuenta de que el uso de una plataforma electrónica, como la que se implementó en este caso, permite construir las bases para un aprendizaje colaborativo. La plataforma permite el intercambio de experiencias, la construcción de trabajos conjuntos y el permanente diálogo en torno al aprendizaje entre pares, así como también la relación con la docente.

Por otra parte, se observa que para el pleno desarrollo de un aprendizaje colaborativo se hace necesario un ambiente en el cual los pares y la docente se involucren en una relación que tenga como uno de sus objetivos el trabajo colaborativo. Este trabajo debiera realizarse durante todo el transcurso de la formación docente, tanto inicial como continua. En este sentido, no es posible afirmar que con el desarrollo de esta experiencia se ha generado una mirada distinta del aprendizaje, aunque sí es posible señalar que el grupo tiene ahora una visión que antes no tenía respecto al trabajo de colaboración y de construcción de redes. 
Otro elemento importante para la construcción de un aprendizaje colaborativo es lo que tiene relación con el ambiente que se genera al interior de la clase. Tanto la docente como los otros compañeros de curso tienen una labor importante a la hora de constituir el ambiente de la clase de un modo más colaborativo, pero no hay dudas de que el peso de esta conducción debe ser de la docente. Ella es quien dirige el curso, la persona encargada de conducir en la línea del apoyo y la colaboración entre pares, por esto es que podemos decir que el aprendizaje colaborativo no solo depende del diseño, tanto curricular como tecnológico, sino que también depende de la dirección, enfoque y motivación desde la docente a cargo.

Es necesario señalar que si no existe un cambio de paradigma completo el aprendizaje colaborativo estará en función de logros limitados como lo es una nota. Los estudiantes no internalizarán el trabajo colaborativo como una manera de resolver los problemas si es que estos no se presentan como reales. Quizás un modo de avanzar en esta perspectiva sea la de generar un modelo de aprendizaje colaborativo basado en situaciones y problemas reales que permita generar una colaboración más allá de la calificación, sino que efectivamente se aprenda y valore el trabajo colaborativo como una manera de aprender.

\section{Bibliografía}

Amigues, R. (1987, septiembre 19-22). Conceptual change and peer interaction. Presentado en: Second European Conference for Research on Learning and Instruction, Tübingen, West-Germany.

Bellefeuille, G. L. (2006). Rethinking reflective practice education in social work education: A blended constructivist and objectivist instructional design strategy for a web-based child welfare practice course. Journal of Social Work Education, 42(I), 85-I03.

Collazos, C.; Guerrero, L. \& Vergara, A. (200I). Aprendizaje Colaborativo: un cambio en el rol del profesor. Presentado en: Tercer Congreso de Educación Superior en Computación, Punta Arenas.

Comisión (2005). Informe comisión sobre formación inicial docente. Santiago: MINEDUC.

Del Valle, G. \& López, M. B. (2005, 29 y 30 de septiembre). Las TIC y 
el trabajo colaborativo en el proceso de enseñanza-aprendizaje en el nivel universitario. Presentado en: Ier Congreso de tecnologías de la información y la comunicación en la enseñanza de las ciencias TICEC05, La Plata, Argentina.

Guldberg, K. \& Pilkington, R. (2006). A community of practice approach to the development of non-traditional learners through networked learning. Journal of Computer Assisted Learning, 22(3), I59-I7I.

Motteram, G. (2006). 'Blended' education and the transformation of teachers: a long-term case study in postgraduate UK Higher Education. British Journal of Educational Technology, 37(I), I7-30.

Mutula, S.; Kalusopa, T.; Moahi, K. \& Wamukoya, J. (2006). Design and implementation of an online information literacy module - Experience of the Department of Library and Information Studies, University of Botswana. Online Information Review, 30(2), I68-I87.

OCDE (2004). Revisión de políticas nacionales de educación. París: OCDE.

Silva-Peña, I.; Borrero, A. M.; Marchant, P.; González, G. \& Novoa, D. (2006). Percepciones de jóvenes acerca del uso de las tecnologías de información en el ámbito escolar. Última Década, 24, 37-60.

Walberg, H. \& Paik, S. (2000). Prácticas eficaces. Serie Prácticas Educativas n³. París: IBE-UNESCO.

Yeh, D.; Lee, C. H. \& Sun, P. C. (2005). The analysis of learning records and learning effect in blended e-learning. Journal of Information Science and Engineering, 2I(5), 973-984. 